\title{
T-Normas, T-Conormas, Complementos e Implicações Intervalares
}

A. TAKAHASHI ${ }^{1}$, B.R.C. BEDREGAL ${ }^{2}$, Departamento de Informática e Matemática Aplicada (DIMAp), Laboratório de Lógica e Inteligência Computacional (LabLIC), Universidade Federal do Rio Grande do Norte (UFRN), Campus Universitário, Lagoa Nova, 59078-970 Natal, RN, Brasil.

Resumo A lógica fuzzy modela matematicamente a imprecisão da linguagem natural, utilizando graus de pertinências (valores entre 0 e 1), contudo, nem sempre é simples especificar com precisão esses graus de pertinências. Existem infinitas formas de generalizar o comportamento dos conectivos lógicos clássicos (álgebra booleana) para valores no conjunto $[0,1]$. As t-normas, t-conormas, implicações e complementos são operações sobre $[0,1]$ satisfazendo certas propriedades que generalizam os conectivos lógicos de conjunção, disjunção, implicação e negação, respectivamente, de forma a preservar algumas das propriedades da lógica clássica desses conectivos.

Este trabalho consiste em introduzir uma generalização de t-norma, t-conorma, implicação e complemento, para o conjunto $\mathbb{I}=\{[a, b]: 0 \leq a \leq b \leq 1\}$, chamados de t-norma intervalar, t-conorma intervalar, implicação intervalar e complemento intervalar, de tal modo que, formas canônicas de se obter t-conorma intervalar, implicação intervalar e complemento intervalar a partir de uma t-norma intervalar sejam preservados.

\section{Introdução}

Na década de 60, Lotfi A. Zadeh em [22] propôs a teoria dos conjuntos fuzzy, cuja principal característica é considerar um grau de pertinência (um valor no intervalo $[0,1])$ para indicar o quanto uma informação pertence a um certo conjunto. Assim, na sua lógica subjacente, isto é, a lógica fuzzy (LF), uma proposição não é simplesmente verdadeira ou falsa, como na lógica clássica, mas pode ter graus de verdade intermediários, tipicamente valores entre 0 (falso absoluto) e 1 (verdade absoluta).

Um sistema computacional inteligente que utiliza lógica fuzzy, sistema fuzzy, é eficiente para tratar com informações incertas [4], onde, as incertezas encontradas na linguagem natural são tratadas por graus de pertinências, ou seja, considerando, por exemplo, um conjunto de pessoas "altas", uma pessoa que possui 1,80 metro de altura e outra que possui 1,78 metro de altura serão consideradas altas, porém, a pessoa que possui 1,80 metro terá um grau de pertinência maior para o grupo

\footnotetext{
${ }^{1}$ adriana@ppgsc.ufrn.br. Bolsista do CNPq - Brasil;

${ }^{2}$ bedregal@dimap.ufrn.br. Parcialmente financiado pelo CNPq, processo 470871/2004-0.
} 
de pessoas "altas" do que a outra pessoa deste conjunto. Contudo, a incorporação do conceito de "grau de verdade" ou grau de pertinência nem sempre é simples de definir, por exemplo, um especialista é capaz de definir o grau de pertinência em uma escala de 0 à 10 para uma determinada instrução, porém, dificilmente será capaz de definir o grau de pertinência para a mesma instrução se for aumentado essa escala para 0 à 100, ou seja, se for exigido uma maior precisão, possivelmente a resposta não seria tão precisa. Uma das alternativas pesquisadas é associar com a matemática intervalar, cujo objetivo é obter um controle automático do erro computacional e conseqüêntemente tratar da imprecisão dos dados [17].

A matemática intervalar tem sido muito utilizada para representar dados incertos e/ou valores qualitativos, seja na computação científica e tecnológica, assim como em inteligência artificial, processamento digital de imagens, entre outros $[1,3,14]$. Assim, a matemática intervalar aliada com a teoria fuzzy permite, em princípio, tratar tanto com a incerteza quanto com a imprecisão, seja no controle automático do erro computacional, tratando da imprecisão dos valores de entrada ou nos erros de arredondamento e/ou truncamento causados durante o processamento computacional $[21,6,11,19,10]$. Para a modelagem da incerteza e imprecisão de uma informação com respeito a uma determinada propriedade tem sido usado subintervalos de $[0,1]$ para atribuir valores verdades às proposições fuzzy. Esta extensão da LF é conhecida como lógica fuzzy valorada intervalarmente ou simplesmente lógica fuzzy intervalar (LFI).

Existem muitas formas de estender os conectivos proposicionais clássicos para o conjunto $[0,1]$, porém, nem sempre essas extensões preservam as propriedades lógicas dos conectivos clássicos. As normas triangulares, ou simplesmente t-normas, foram introduzidas por Menger [16] e, Schweizer e Skar [18] deram uma axiomática para as t-normas. A noção de t-normas e t-conormas conhecidas foram modeladas à partir dos conectivos de conjunção e disjunção, respectivamente. Outros conectivos proposicionais fuzzy são gerados através desses axiomas, desde que, exista uma ligação forte entre esses conectivos proposicionais fuzzy, sendo possível obter de forma canônica uma implicação fuzzy à partir de uma t-norma, ou uma negação fuzzy à partir de uma implicação [5].

Várias generizações de t-normas para conjuntos intervalares são encontradas na literatura $[8,23]$. Neste trabalho, foi generalizado a noção de t-conormas, negação fuzzy e implicação fuzzy para a representação da teoria intervalar, e também foi introduzido a forma canônica de obter os conectivos fuzzy para a versão intervalar, mostrando que esses conectivos preservam as propriedades dos conectivos fuzzy.

\section{T-normas e T-normas Intervalares}

A norma triangular, ou também conhecida como t-norma, é uma operação binária utilizada geralmente para representar o operador and, ou a intersecção.

Definição 2.1 (T-norma). Uma t-norma é uma função $T:[0,1]^{2} \rightarrow[0,1]$ que é: Simétrica, Associativa, Monotônica e 1 é um elemento neutro $[13,20]$.

Seja $\mathbb{I}[0,1]=\{[a, b] \quad \mid \quad 0 \leq a \leq b \leq 1\}$ o conjunto dos intervalos entre 0 e 1. Será usado por convenção que $X \in \mathbb{I}[0,1]$ então $X=\left[x_{i}, x_{s}\right]$. Sendo possível definir 
diversas ordens parciais em $\mathbb{I}[0,1]$. As duas mais conhecidas são a dada por Kulisch e Miranker em [12]:

$$
X \leq Y \text { se e somente se } x_{i} \leq y_{i} \text { e } x_{s} \leq y_{s}
$$

e a ordem de inclusão dada por Moore em [17]:

$$
X \subseteq Y \text { se e somente se } y_{i} \leq x_{i} \text { e } x_{s} \leq y_{s} .
$$

Seja $\Delta \subseteq \mathbb{I}[0,1]$. O supremo de $\Delta$, com respeito à ordem de Kulisch-Miranker é

$$
\sup (\Delta)=\left[\sup \left\{x_{i} \mid X \in \Delta\right\}, \sup \left\{x_{s} \mid X \in \Delta\right\}\right] .
$$

Os especialistas podem descrever suas incertezas com graus de pertinências precisos, mas como dizer, por exemplo, que o grau de pertinência de uma pessoa pertence ao conjunto dos altos é $\sqrt{0.2}$. Assim, o mais correto seria usar intervalos como graus de pertinências. Portanto, a noção de t-norma deve ser estendida para intervalos.

Definição 2.2 (T-norma intervalar). Uma função IT $: \mathbb{I}[0,1]^{2} \rightarrow \mathbb{I}[0,1]$ é uma tnorma intervalar se IT for simétrica, associativa, monotônica com respeito à ordem de Kulisch-Miranker e à inclusão, e [1,1] é um elemento neutro.

Proposição 2.1. Se $T:[0,1]^{2} \rightarrow[0,1]$ é uma t-norma real, então $I[T]: \mathbb{I}[0,1]^{2} \rightarrow$ $\mathbb{I}[0,1]$ definida por

$$
I[T](X, Y)=\left[T\left(x_{i}, y_{i}\right), T\left(x_{s}, y_{s}\right)\right] .
$$

é uma t-norma intervalar, denominada de t-norma intervalar derivada de $T$.

Demonstração: Por definição de intervalo, $x_{i} \leq x_{s}$ e $y_{i} \leq y_{s}$, então pela monotonicidade de $T$, temos que $T\left(x_{i}, y_{i}\right) \leq T\left(x_{s}, y_{s}\right)$. Logo, $I[T]$ está bem definida.

A seguir será provado que $I[T]$ é uma t-norma intervalar, ou seja, que satisfaz as seguintes propriedades:

Simétrica: $\forall X, Y \in \mathbb{I}[0,1]$

$$
\begin{aligned}
I[T](X, Y) & =\left[T\left(x_{i}, y_{i}\right), T\left(x_{s}, y_{s}\right)\right] \\
& =\left[T\left(y_{i}, x_{i}\right), T\left(y_{s}, x_{s}\right)\right] \\
& =I[T](Y, X) .
\end{aligned}
$$

Associativa: $\forall X, Y, Z \in \mathbb{I}[0,1]$

$$
\begin{aligned}
I[T](X, I[T](Y, Z)) & =I[T]\left(X,\left[T\left(y_{i}, z_{i}\right), T\left(y_{s}, z_{s}\right)\right]\right) \\
& =\left[T\left(x_{i}, T\left(y_{i}, z_{i}\right)\right), T\left(x_{s}, T\left(y_{s}, z_{s}\right)\right)\right] \\
& =\left[T\left(T\left(x_{i}, y_{i}\right), z_{i}\right), T\left(T\left(x_{s}, y_{s}\right), z_{s}\right)\right] \\
& =I[T]\left(\left[T\left(x_{i}, y_{i}\right), T\left(x_{s}, y_{s}\right)\right], Z\right) \\
& =I[T](I[T](X, Y), Z) .
\end{aligned}
$$

Monotônica: Se $X \leq Z$ e $Y \leq W$ então $x_{i} \leq z_{i}, x_{s} \leq z_{s}, y_{i} \leq w_{i}$ e $y_{s} \leq w_{s}$. Logo, $T\left(x_{i}, y_{i}\right) \leq T\left(z_{i}, w_{i}\right)$ e $T\left(x_{s}, y_{s}\right) \leq T\left(z_{s}, w_{s}\right)$. Assim, $\left[T\left(x_{i}, y_{i}\right), T\left(x_{s}, y_{s}\right)\right] \leq$ $\left[T\left(z_{i}, w_{i}\right), T\left(z_{s}, w_{s}\right)\right]$ e portanto $I[T](X, Y) \leq I[T](Z, W)$. 
Inclusão monotônica: Se $X \subseteq Z$ e $Y \subseteq W$, então $z_{i} \leq x_{i} \leq x_{s} \leq z_{s}$ e $w_{i} \leq y_{i} \leq$ $y_{s} \leq w_{s}$. Por definição, $I[T](X, Y)=\left[T\left(x_{i}, y_{i}\right), T\left(x_{s}, y_{s}\right)\right]$ e $I[T](Z, W)=$ $\left[T\left(z_{i}, w_{i}\right), T\left(z_{s}, w_{s}\right)\right]$. Logo, por monotonicidade de $T, T\left(z_{i}, w_{i}\right) \leq T\left(x_{i}, y_{i}\right) \leq$ $T\left(x_{s}, y_{s}\right) \leq T\left(z_{s}, w_{s}\right)$. Portanto, $I[T](X, Y) \subseteq I[T](Z, W)$.

Elemento Neutro: $\forall X \in \mathbb{I}[0,1]$,

$$
\begin{aligned}
I[T](X,[1,1]) & =\left[T\left(x_{i}, 1\right), T\left(x_{s}, 1\right)\right] \\
& =\left[x_{i}, x_{s}\right] \\
& =X .
\end{aligned}
$$

\section{T-conormas e T-conormas Intervalares}

Uma t-conorma é uma implementação da união, ou do operador or.

Definição 3.1 (T-conorma). Uma conorma triangular (t-conorma ou co-norma), é uma função $S:[0,1]^{2} \rightarrow[0,1]$ que é: Simétrica, Associativa, Monotônica e 0 é um elemento neutro [13, 20]:

Seja $T$ uma t-norma, então,

$$
S_{T}(x, y)=1-T(1-x, 1-y)
$$

é uma t-conorma, denominada de t-conorma derivada de $T$.

A t-conorma intervalar é uma extensão da t-conorma.

Definição 3.2 (T-conorma intervalar). Uma função $I S: \mathbb{I}[0,1]^{2} \rightarrow \mathbb{I}[0,1]$ é uma t-conorma intervalar se IS for simétrica, associativa, monotônica, e $[0,0]$ é um elemento neutro.

Proposição 3.1. Se $S:[0,1]^{2} \rightarrow[0,1]$ é uma t-conorma, então $I[S]: \mathbb{I}[0,1]^{2} \rightarrow$ $\mathbb{I}[0,1]$ definida por

$$
I[S](X, Y)=\left[S\left(x_{i}, y_{i}\right), S\left(x_{s}, y_{s}\right)\right]
$$

é uma t-conorma intervalar, denominada de t-conorma intervalar derivada da $t$ conorma $S$.

Demonstração: Análoga à prova da proposição 2.1.

Proposição 3.2. Seja IT uma t-norma intervalar. Então $S_{I T}: \mathbb{I}[0,1]^{2} \rightarrow \mathbb{I}[0,1]$ definida por

$$
S_{I T}(X, Y)=[1,1]-I T([1,1]-X,[1,1]-Y)
$$

é uma t-conorma intervalar, denominada de t-conorma intervalar derivada de IT

Demonstração: A seguir, será provado que $S_{I T}$ satisfaz as seguintes propriedades de t-conorma intervalar. 
Simétrica: $\forall X, Y \in \mathbb{I}[0,1]$

$$
\begin{aligned}
S_{I T}(X, Y) & =[1,1]-I T([1,1]-X,[1,1]-Y) \\
& =[1,1]-I T([1,1]-Y,[1,1]-X) \\
& =S_{I T}(Y, X) .
\end{aligned}
$$

Associativa: $\forall X, Y, Z \in \mathbb{I}[0,1]$

$$
\begin{aligned}
S_{I T}\left(X, S_{I T}(Y, Z)\right) & =S_{I T}(X,[1,1]-I T([1,1]-Y,[1,1]-Z)) \\
& =[1,1]-I T([1,1]-X,[1,1]-([1,1]-I T([1,1]-Y,[1,1]-Z))) \\
& =[1,1]-I T([1,1]-X, I T([1,1]-Y,[1,1]-Z)) \\
& =[1,1]-I T(I T([1,1]-X,[1,1]-Y),[1,1]-Z) \\
& =[1,1]-I T([1,1]-([1,1]-I T([1,1]-X,[1,1]-Y)),[1,1]-Z) \\
& =S_{I T}([1,1]-I T([1,1]-X,[1,1]-Y), Z) \\
& =S_{I T}\left(S_{I T}(X, Y), Z\right) .
\end{aligned}
$$

Monotônica: Se $X \leq Z$ e $Y \leq W$ então $[1,1]-X \leq[1,1]-Z$ e $[1,1]-Y \leq$ $[1,1]-W$. Logo, por monotonicidade de $I T, I T([1,1]-X,[1,1]-Y) \leq$ $I T([1,1]-Z,[1,1]-W)$. Portanto, $[1,1]-I T([1,1]-X,[1,1]-Y) \leq[1,1]-$ $I T([1,1]-Z,[1,1]-W)$. Assim, $S_{I T}(X, Y) \leq S_{I T}(Z, W)$.

Inclusão monotônica: Se Se $X \subseteq Z$ e $Y \subseteq W$ então $[1,1]-X \subseteq[1,1]-Z$ e $[1,1]-Y \subseteq[1,1]-W$. Logo, por monotonicidade de $I T, I T([1,1]-X,[1,1]-$ $Y) \subseteq I T([1,1]-Z,[1,1]-W)$. Portanto, $[1,1]-I T([1,1]-X,[1,1]-Y) \subseteq$ $[1,1]-I T([1,1]-Z,[1,1]-W)$. Assim, $S_{I T}(X, Y) \subseteq S_{I T}(Z, W)$.

Elemento Neutro: $\forall X \in \mathbb{I}[0,1]$,

$$
\begin{aligned}
S_{I T}(X,[0,0]) & =[1,1]-I T([1,1]-X,[1,1]-[0,0]) \\
& =[1,1]-I T([1,1]-X,[1,1]) \\
& =[1,1]-([1,1]-X) \\
& =X .
\end{aligned}
$$

A seguir será provado que as construções de t-conormas intervalares à partir de t-conormas são derivadas de uma t-norma, corresponde com a de t-conorma intervalar derivada da t-norma intervalar obtida à partir da t-norma. Ou seja, que o seguinte diagrama

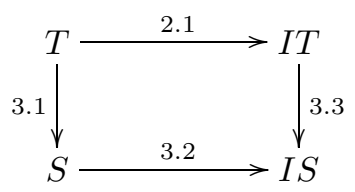

comuta.

Teorema 3.1. Seja $T$ uma t-norma real, então $I\left[S_{T}\right]=S_{I[T]}$. 


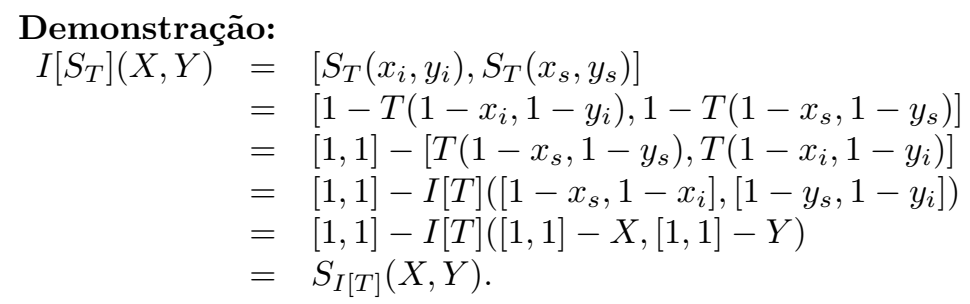

\section{Implicação e Implicação intervalar}

O operador de implicação é utilizado para modelar regras de inferência do tipo se $<$ premissa $>$ então $<$ conclusão $>$.

Definição 4.1 (Implicação). Uma função $P:[0,1]^{2} \rightarrow[0,1]$ é uma função de implicação se a função satisfaz as seguintes propriedades, ver em $[2,13,5,9,7]$ :

P1: Se $x \leq z$ implica que $P(x, y) \geq P(z, y), \forall x, y, z \in[0,1]$.

P2: Se $y \leq z$ implica que $P(x, y) \leq P(x, z), \forall x, y, z \in[0,1]$.

P3: $P(0, y)=1, \forall y \in[0,1]$.

P4: $P(x, 1)=1, \forall y \in[0,1]$.

P5: $P(1,0)=0$.

Seja $T$ uma t-norma. Então a função definida por

$$
P_{T}(x, y)=\sup \{z \mid T(x, z) \leq y\}, \forall x, y, z \in[0,1]
$$

é uma implicação, conhecida como R-implicação associada a $T$ ou residuo de $T$.

Definição 4.2 (Implicação intervalar). Uma função IP $: \mathbb{I}[0,1]^{2} \rightarrow \mathbb{I}[0,1]$ é uma implicação intervalar se $\forall X, Y, Z \in \mathbb{I}[0,1]$, IP satisfazer as propriedades:

IP1: Se $X \leq Z$ então $I P(X, Y) \geq I P(Z, Y)$.

IP2: Se $Y \leq Z$ então $I P(X, Y) \leq I P(X, Z)$.

IP3: Se $W \subseteq Y$ e $X \subseteq Z$ então $I P(W, X) \supseteq I P(Y, Z)$.

IP4: $\operatorname{IP}([0,0], Y)=[1,1]$.

IP5: $\operatorname{IP}(X,[1,1])=[1,1]$.

IP6: IP $([1,1],[0,0])=[0,0]$. 
Proposição 4.1. Se $P:[0,1]^{2} \rightarrow[0,1]$ é uma implicação real, então $I[P]:$ $\mathbb{I}[0,1]^{2} \rightarrow \mathbb{I}[0,1]$ definida por

$$
I[P](X, Y)=\left[P\left(x_{s}, y_{i}\right), P\left(x_{i}, y_{s}\right)\right]
$$

é uma implicação intervalar, denominada de implicação intervalar derivada da implicação $P$.

Demonstração: Por definição de intervalo, $\operatorname{IP}(X, Y)$ está bem definida, pois, $P\left(x_{s} \leq y_{i}\right) \leq P\left(x_{i} \leq x_{i}\right) \leq P\left(x_{i} \leq y_{s}\right)$.

Sejam $X, Y, Z, \in I[0,1]$.

IP1: Se $X \leq Z$ então, por P1, $P\left(x_{s}, y_{i}\right) \geq P\left(z_{s}, y_{i}\right)$ e $P\left(x_{i}, y_{s}\right) \geq P\left(z_{i}, y_{s}\right)$ então $I[P](X, Y) \geq I[P](Z, Y)$.

IP2: Se $Y \leq Z$ então, por P2, $P\left(x_{s}, y_{i}\right) \leq P\left(x_{s}, z_{i}\right)$ e $P\left(x_{i}, y_{s}\right) \leq P\left(x_{i}, z_{s}\right)$ então $I[P](X, Y) \leq I[P](Z, Y)$.

IP3: Se $W \subseteq Y$ e $X \subseteq Z$ então, por P2, $P\left(w_{s}, x_{i}\right) \geq P\left(w_{s}, z_{i}\right)$ e por P1, $P\left(w_{s}, z_{i}\right) \geq P\left(y_{s}, z_{i}\right)$. Logo, $\left.P\left(w_{s}, x_{i}\right) \geq P\left(y_{s}, z_{i}\right)\right]$. Analogamente, por P2, $P\left(w_{i}, x_{s}\right) \leq P\left(w_{i}, z_{s}\right)$ e por P1, $P\left(w_{i}, z_{s}\right) \geq P\left(y_{i}, z_{s}\right)$. Então, $I[P](W, X) \subseteq$ $I[P](Y, Z)$.

IP4: $I[P]([0,0], Y)=\left[P\left(0, y_{i}\right), P\left(0, y_{s}\right)\right]=[1,1]$.

IP5: $I[P](X,[1,1])=\left[P\left(x_{s}, 1\right), P\left(x_{i}, 1\right)\right]=[1,1]$.

IP6: $I[P]([1,1],[0,0])=[P(1,0), P(1,0)]=[0,0]$.

Proposição 4.2. Seja IT uma t-norma intervalar. Então $I P_{I T}: I[0,1]^{2} \rightarrow I[0,1]$ definida por

$$
I P_{I T}(X, Y)=\sup \{Z \in I[0,1] \mid I T(X, Z) \leq Y\}
$$

é uma implicação intervalar.

A seguir será provado que as construções implicação intervalar a partir de implicações derivadas de uma t-norma, corresponde com a de implicação intervalar derivada da t-norma intervalar obtida a partir da t-norma. Ou seja, que o seguinte diagrama

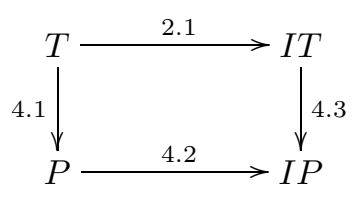

comuta.

Teorema 4.1. Seja T uma t-norma real, então $I\left[P_{T}\right]=P_{I[T]}$ 
Demonstração: Note que claramente o conjunto $\left\{Z \in \mathbb{I}[0,1] \mid\left[T\left(x_{i}, z_{i}\right), T\left(x_{s}, z_{s}\right)\right]\right.$ $\leq Y\}$ é dirigido com respeito à ordem de Kulisch-Mikranker. Assim,

$$
\begin{aligned}
P_{I[T]}(X, Y) & =\sup \{Z \in \mathbb{I}[0,1] \mid I[T](X, Z) \leq Y\} \\
& =\sup \left\{Z \in \mathbb{I}[0,1] \mid\left[T\left(x_{i}, z_{i}\right), T\left(x_{s}, z_{s}\right)\right] \leq Y\right\} \\
& =\left[\sup \left\{z \in[0,1] \mid T\left(x_{s}, z\right) \leq y_{i}\right\}, \sup \left\{z \in[0,1] \mid T\left(x_{i}, z\right) \leq y_{s}\right\}\right] \\
& \left.=\left[P_{T}\left(x_{i}, y_{i}\right), P_{(} x_{s}, y_{s}\right)\right] \\
& =I\left[P_{T}\right] .
\end{aligned}
$$

\section{Complemento e Complemento Intervalar}

Na teoria dos conjuntos fuzzy temos o operador de complemento, ou negação fuzzy, para interpretar o operador lógico de negação.

Definição 5.1 (Complemento). Uma função $C:[0,1] \rightarrow[0,1]$ é um complemento se a função satisfaz as seguintes propriedades:

$C 1: C(0)=1, C(1)=0$.

C2: Se $x \geq y$ então $C(x) \leq C(y), \forall x, y \in[0,1]$.

Se C também satisfaz:

C3: $C(C(x))=x, \forall x \in[0,1]$.

então é chamado de complemento forte.

O complemento intervalar é uma derivação do complemento dos conjuntos fuzzy, satisfazendo suas propriedades e também da teoria intervalar.

Definição 5.2 (Complemento intervalar). Uma função $I C: \mathbb{I}[0,1] \rightarrow \mathbb{I}[0,1]$ é um complemento intervalar se,

IC1: $\operatorname{IC}([0,0])=[1,1]$ e $\operatorname{IC}([1,1])=[0,0]$.

IC2: Se $X \geq Y$ então $I C(X) \leq I C(Y), \forall X, Y \in I[0,1]$.

IC3: Se $X \subseteq Y$ então $I C(X) \subseteq I C(Y), \forall X, Y \in I[0,1]$.

Um complemento intervalar IC é forte, se

IC4: $I C(I C(X))=X, \forall X \in I[0,1]$.

Proposição 5.1. Se $C:[0,1] \rightarrow[0,1]$ é um complemento, então $I C: I[0,1] \rightarrow$ $I[0,1]$ definida por

$$
I[C](X)=\left[C\left(x_{s}\right), C\left(x_{i}\right)\right]
$$

é um complemento intervalar, denominada de complemento intervalar derivado do complemento $C$. Se $C$ é um complemento forte então $I[C]$ é um complemento intervalar forte.

Demonstração: Segue o mesmo formato que a prova da proposição 2.1. 


\section{Conclusões}

Neste trabalho, foi apresentado extensões intervalares dos modelos fuzzy para os operadores lógicos de conjunção, disjunção, implicação e negação. Ainda, foi mostrado como as formas canônicas de se obter alguns desses operadores à partir da t-norma são preservados pela construção intervalar.

A extensão proposta aqui para t-normas é diferente de outras generalizações de t-normas para intervalos, tais como as definida por [23, 8]. A primeira exige além destas propriedades que a função seja contínua e estritamente monotônica com respeito à ordem de Kulisch-Miranker, com o qual, nem toda t-norma real teria uma t-norma intervalar que estendesse ela. A segunda exige que $\operatorname{IT}\left([0,1],\left[x_{i}, x_{s}\right]\right)=$ $\left[0, x_{s}\right]$ e não requer que satisfaça a inclusão monotônica, entre outras propriedades. Nesse trabalho, cada t-norma intervalar tem associado uma única t-norma, porém, nem toda t-norma tem associada uma t-norma intervalar, com o qual, não é possível generalizar intervalarmente todas as interpretações fuzzy dos conectivos lógicos.

Assim, as generalizações aqui apresentadas demarcam o início de um estudo sobre lógica fuzzy intervalar no sentido estreito [15], na qual as construções e conceitos usuais para o caso pontual sejam estendidas, preservando o máximo possível, as relações entre eles.

\section{Referências}

[1] M.S. Aguiar, A.C.R. Costa e G.P. Dimuro, ICTM: An interval tessellationbased model for reliable topographic segmentation. Numerical Algorithms, $\mathbf{3 6}$ (2004), 1-10.

[2] M. Baczynski, Residual implications revisited. notes on the Smets-Magrez. Fuzzy Sets and Systems, 145, No. 2 (2004), 267-277.

[3] L.V. Barboza, G.P. Dimuro e R.H.S. Reiser, Power flow with load uncertainty. TEMA - Tendências em Matemática Aplicada e Computacional, 5 (2004), 2736 .

[4] G. Bojadziev e M. Bojadziev, "Fuzzy Sets, Fuzzy Logic, Applications", volume 5. World Scientific, 1995.

[5] H. Bustince, P. Burilo e F. Soria, Automorphism, negations and implication operators. Fuzzy Sets and Systems, 134 (2003), 209-229.

[6] D. Dubois e H. Prade, Random sets and fuzzy interval analysis. Fuzzy Sets and Systems, 42 (1991), 87-101.

[7] J.C. Fodor, On fuzzy implication operators. Fuzzy Sets and Systems, 42 (1991), 293-300.

[8] M. Gehrke, C. Walker e E. Walker, Algebraic aspects of fuzzy sets and fuzzy logic. Proceedings of Workshop on Current Trends and Develoments in Fuzzy Logic, pp. 101-170, 1999. 
[9] R. Horcik e M. Navara, Validation sets in fuzzy logics. Kybernetika, 38 No. 3 (2002), 319-326.

[10] L.J. Kohout e E. Kim, Characterization ofinterval fuzzy logic systems of connectives by group transformation. Reliable Computing, 10 (2004), 299-334.

[11] V. Kreinovich e M. Mukaidono, Interval (pairs of fuzzy values), triples, etc.: Can we thus get an arbitrary ordering? Proceedings of the $9^{\text {th }}$ IEEE International Conference on Fuzzy Systems. San Antonio, Texas, 1 (2000), 234-238.

[12] U.B. Kulisch e W.L. Miranker, "Computer Arithmetic Theory and Pratice", Academic Press, San Diego, 1981.

[13] J.M. Leski, Insensitive learning techniques for approximate reasoning system. Int. J. Computational Cognition, 1, No. 1 (2003), 21-77.

[14] A. Lyra, B.R.C. Bedregal, R. Callejas-Bedregal e A.D. Doria Neto, The interval digital images processing. WSEAS Transactions on Circuits and Systems, 3, No. 2 (2004), 229-233.

[15] R.J. Marks-II, "Fuzzy logic technology and applications", chapter Preface by L.A. Zadeh. IEEE Technical Activities Board, 1994.

[16] K. Menger, Statistical metrics. Proc. Nat. Acad., pp. 535-537, 1942.

[17] R.E. Moore, Methods and Applications of Interval Arithmetic. PhD thesis, Studies in Applied Mathematics - SIAM, 1979.

[18] B. Schweizer e A. Sklar, Associative functions and statistical triangle inequalities. Publicationes Mathematicae Debrecen, pp. 168-186, 1961.

[19] M.M.M.T. Silveira e B.R.C. Bedregal, A method of inference and defuzzyfication fuzzy interval. Proceeding of the IASTED International Conference on Artificial Intelligence e Applications, pp. 242-247, 2001.

[20] L.H. Tsoukalas e R.E. Uhrig, Fuzzy e Neural Approaches in Engineering. Wiley Interscience, 1997.

[21] I.B. Turksen, Interval valued fuzzy sets based on normal forms. Fuzzy Sets and Systems, 20 (1986), 191-210.

[22] L.A. Zadeh, Fuzzy sets. Proc. Nat. Acad., pp. 535-537, 1942.

[23] Q. Zuo, Description of strictly monotonic interval AND/OR operations. APIC'S Proceedings: International Workshop on Applications of Interval Computations, pp. 232-235, 1995. 